\title{
DOUBLE GAUSS BASED UNSUPERVISED SCORE NORMALIZATION IN SPEAKER VERIFICATION
}

\author{
Wu Guo, Li-Rong Dai, Ren-Hua Wang
iFly Speech Lab, Department of Electronic Engineering and Information Science, University of Science and Technology of China, Hefei
\{guowu,lrdai,rhw\}@ustc.edu.cn

\begin{abstract}
In text-independent speaker verification, unsupervised mode can improve system performance. In traditional systems, the speaker model is updated when a test speech has a score higher than a particular threshold; we call this unsupervised model training. In this paper, an unsupervised score normalization is proposed. A target speaker score Gauss and an impostor score Gauss are set up as a prior; the parameters of the impostor score model are updated using the test score. Then the test score is normalized by the new impostor score model. When the unsupervised score normalization, unsupervised model training and factor analysis are adopted in the NIST 2006 SRE core test, the EER of the system is $4.29 \%$.
\end{abstract}

Index Terms - speaker verification, factor analysis, unsupervised mode

\section{INTRODUCTION}

In the recent NIST speaker recognition evaluation (SRE) [1], unsupervised mode is allowed. The participants can use the previous test speech to update speaker models. In most cases [2,3], the researchers set up a threshold. When the test speech score is higher than the threshold, they use the test speech to update the GMM or SVM models' parameters. In [4], the maximum a posterior (MAP) algorithm is adopted to update the speaker models. At the occurrence of a new test utterance, this utterance is used to update all the speaker models. This process can be defined as unsupervised model training. Although unsupervised model training can improve the system performance, this algorithm needs to re-train the speaker model with the test utterance. It is a time-consuming process.

Score normalization can be addressed to reduce the effects of both speaker-dependent and speaker-independent modifications on a speech signal. The most commonly used normalization algorithms are zero normalization (Znorm) and test normalization (Tnorm)[5]. Znorm attempts to align intra-speaker differences of imposter scores' distributions. In practice, a speaker model is tested against a set of speech uttered by some impostors, resulting in an impostor similarity score distribution. Speaker-dependent mean and variance can be estimated from the distribution. Tnorm attempts to remove inter-speakers bias of imposter scores distributions. In Tnorm, the incoming speech signal is traditionally compared with claimed speaker model as well as with a set of impostor models to estimate impostor score distribution and normalization parameters consecutively. So Tnorm differs from Znorm by the use of impostor models instead of test speech signals.

In NIST SRE 2006, each speaker model is tested by a set of test utterances. The sequence of the trials is predefined for unsupervised mode. Participants cannot use other speaker model information. This process is very similar to the Znorm process. So we can use previous test scores to update the Znorm parameters dynamically. We call this process the unsupervised score normalization. When the unsupervised score normalization is addressed, the system performance can improve significantly. Furthermore, unsupervised score normalization can be integrated with unsupervised model training to further improve recognition performance. .

The factor analysis $[6,7,8]$ has been found to be very effective in reducing the channel bias in the Gaussian mixture models and universal background models (GMMUBM) system[6]. In this paper, we use factor analysis to reduce channel bias. Compared with the traditional GMMUBM system, the score normalization process will greatly improve the system recognition rate after factor analysis.

The remainder of this paper is organized as follows. In section 2 we describe the factor analysis algorithm used in this paper. The unsupervised score normalization is discussed in Section 3. In section 4 we present experiments and results of the proposed algorithms using the NIST SRE 2006 core test corpora. Finally, conclusion is given in Section 5 . 


\section{FACTOR ANALYSIS}

In the GMM_UBM framework, the speaker model $\lambda$ is depicted as:

$$
p(\boldsymbol{x} \mid \lambda)=\sum_{c=1}^{M} w_{c} p_{c}\left(\boldsymbol{x} ; \boldsymbol{m}_{c}, \Sigma_{c}\right)
$$

The subscript $c$ indicates the component of Gaussian mixture and $M$ is the Gaussian mixture order. If we stack all the mean vectors $\boldsymbol{m}_{c}$ of the GMM to form a vector $\boldsymbol{m}$, this $\boldsymbol{m}$ is the GMM supervector. The session-dependent supervector $\boldsymbol{m}_{h}(s)$ can be represented by (2), where $h$ is the session indication.

$$
\boldsymbol{m}_{h}(s)=\boldsymbol{m}+\boldsymbol{y}(s)+\boldsymbol{U} \boldsymbol{x}_{h}(s)
$$

$\boldsymbol{m}$ is the supervector of the UBM and $\boldsymbol{y}(s)$ is the speaker supervector offset from the UBM. The dimension of vectors $\boldsymbol{m}_{h}(s), \boldsymbol{m}$ and $\boldsymbol{y}(s)$ is $M D \times 1$, and $D$ is the dimension of acoustic feature. $\boldsymbol{U} \boldsymbol{x}_{h}(s)$ represents session offset. $\boldsymbol{U}$ is a $M D$ $\times R_{c}$ matrix that represents the channel space, $x_{h}(s)$ is a $R_{c}$ $\times 1$ channel factor. $R_{c}$ is the number of channel factors. Averagely, the value of $R_{c}$ is 30-50. The calculation of $\boldsymbol{U}$, $\boldsymbol{y}(s), \boldsymbol{x}_{h}(s)$ and its usage in speaker recognition can be found in $[6,8]$.

\section{THE UNSUPERVISED SCORE NORMALIZATION}

The Znorm parameters are estimated from scores derived by scoring a set of imposter utterances through each speaker model. We can use the previous test scores of the same claimed speaker to update the normalization parameters. However, in the test process, some scores belong to the target speaker and some belong to the impostors. We should only use the impostors' score to update the normalization parameters. It is important to judge whether a score belongs to the impostors or not. A score threshold can be set up to finish this work, but its performance can be improved. We set up two score Gaussian models in this paper. If we can produce a development corpus similar to the test corpus, we can obtain the target speakers' scores and imposters' scores for all the test utterances. These two scores distributions can form two Gaussian models: $\Gamma_{\text {impostor }}$ and $\Gamma_{\text {target }}$. The mean and variance $\left\{\mu_{\mathrm{imp}}, \delta_{\mathrm{imp}}\right\},\left\{\mu_{\mathrm{tar}}, \delta_{\mathrm{tar}}\right\}$ of these two Gaussian models can be calculated. These two Gaussian models are addressed as a prior score distributions of the impostor and target speaker. These two score distributions are depicted in Figure 1.

In each speaker test process, we can use some utterance to get the imposters score distribution $\Lambda_{\text {impostor }}$ of this speaker, this process is the same as the Znorm. The mean and variance of $\Lambda_{\text {impostor }}$ is $\left\{\tilde{\mu}_{i m p}, \tilde{\delta}_{i m p}\right\}$. The target speaker score distribution $\Lambda_{\text {target }}\left\{\tilde{\mu}_{\text {tar }}, \tilde{\delta}_{\text {tar }}\right\}$ can be deduced using equation (3). This process is depicted in Figure 2.

$$
\begin{aligned}
& \tilde{\mu}_{t a r}=\tilde{\mu}_{i m p}+\frac{\tilde{\delta}_{i m p}}{\delta_{i m p}}\left(\mu_{t a r}-\mu_{i m p}\right) \\
& \tilde{\delta}_{t a r}=\frac{\delta_{t a r}}{\delta_{i m p}} \tilde{\delta}_{i m p}
\end{aligned}
$$

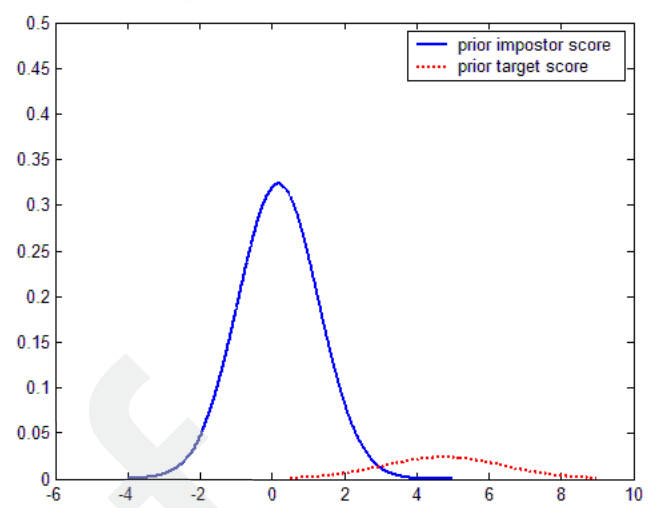

Fig 1: The impostor and target score distributions in development corpus.

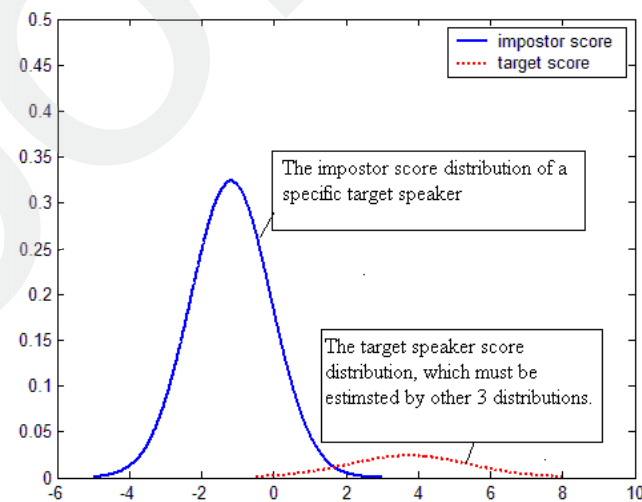

Fig 2: The speaker-dependent impostor and target score distributions.

In the test process, each utterance can produce a score $S$. We use (4) to determine the probabilistic alignment of this score into the two score Gauss $\Lambda_{\text {impostor }}$ and $\Lambda_{\text {target }}$.

$$
\begin{aligned}
& P_{\text {target }}=\frac{p\left(S \mid \Lambda_{\text {target }}\right)}{p\left(S \mid \Lambda_{\text {target }}\right)+p\left(S \mid \Lambda_{\text {impostor }}\right)} \\
& P_{\text {impostor }}=1-P_{\text {target }}
\end{aligned}
$$

$p\left(S \mid \Lambda_{\text {impostor }}\right)$ and $p\left(S \mid \Lambda_{\text {target }}\right)$ are the Gaussian probabilities. The mean of $\Lambda_{\text {impostor }}$ can be updated using equation (5), which is the standard MAP process. Because there is only one score for each time, it is unreasonable to update the variance of $\Lambda_{\text {impostor. }} \tau$ is the relevance factor. In our experiments, we set $\tau=48$.

$$
\tilde{\mu}_{\text {imp }}^{(\text {new })}=\frac{P_{\text {impostor }}}{P_{\text {impostor }}+\tau} S+\frac{\tau}{P_{\text {impostor }}+\tau} \tilde{\mu}_{\text {imp }}^{(\text {old })}
$$


Finally, the score of next test utterance is normalized by (6), just as Znorm.

$$
\tilde{S}=\frac{S-\tilde{\mu}_{i m p}^{(n e w)}}{\tilde{\delta}_{i m p}}
$$

\section{EXPERIMENTS}

Speaker verification experiments are carried out on the NIST SRE 20061 con4w-1con4w corpus (core test). This corpus consists of 810 target speaker models. There are 51448 trials for all 810 speaker models. Each train and test conversation has an average duration of 5 minutes, with 2.5 minutes of speech on average after silence removal. Although there are speakers of both genders in the corpus, no cross-gender trials are defined.

The NIST SRE 2004 corpus is selected to train channel space $\boldsymbol{U}$ in (2). The NIST SRE 2004 corpus is a multilanguage channel balance corpus. It is very good for UBM model training and channel matrix training. There are 310 speakers in NIST 2004 corpus. We only select utterances longer than 30 seconds from the original recording. As a result, 5450 utterances are selected from 310 subjects.

The NIST SRE 2004 1side-train corpus is adopted as the Tnorm corpus. The NIST SRE 2005 1side-train corpus is adopted as the Znorm corpus.

\subsection{Front end processing}

We use MFCC acoustic features in our experiments. The speech is pre-emphasized with a factor of 0.97 and segmented into frames by a $20-\mathrm{ms}$ Hamming window shifting at a 10-ms frame rate. Each speech signal is parameterized using the first 13 MFCCs, and their first and second derivatives, forming a 39-dimension feature vector. RASTA filtering is used to remove linear channel convolution effects. Energy based VAD detector is addressed to discard silence frames from data files. The CMS and Gaussianization is applied to all the MFCCs.

\subsection{Channel space training}

As there are no cross-gender trials, the female and male trials are processed gender-dependently. The NIST SRE 2004 1side corpus is used to train the UBM models. The GMM of UBMs is 512 .

The channel space for female and male are trained gender-dependently. The NIST SRE 2004 corpus is used to train the channel space matrix $\boldsymbol{U}$. After 6 iterations, we can get a stable channel matrix $\boldsymbol{U}$. The factor number is 30 . In this paper, we use almost the same algorithm as [8].

\subsection{System description}

We set up seven systems in our experimrnts. The first system is the standard GMM-UBM. Systems 2 to 7 are factor analysis based GMM-UBM systems. These systems are as following.

(1) The first system is the standard GMM-UBM. This system is same with the system in [9]. The number of GMM is 2048. The MAP is adopted to train the speaker model. Tnorm is adopted to improve the performance.

(2).The second system is the factor analysis based GMM-UBM system. The number of GMM is 512 .

(3).The third system is almost same as the second system except for TZnorm.

(4).The difference between the fourth system and the third system is that the unsupervised score normalization is used to replace the Znorm algorithm in the third system. In the unsupervised score normalization, we use equation (4) to align the probabilities of the score against the two score models.

(5).In system 5, we does not use equation (4) to align the probabilities. Instead, we set a threshold 3.5. In test process, the log-likelihood ratio of the test score is first processed by Tnorm. If the value of the score is below the threshold, the $P_{\text {impostor }}$ in (5) is set to 1 ; and equation (5) is addressed to update the parameters of the impostor score distribution. If the score is larger than the threshold, the parameters of the impostor score distribution will not be updated.

(6).System 6 is used to compare with system 4 . The algorithm in [2] is addressed in our unsupervised model training process. We also set threshold 3.5. In test process, the log-likelihood ratio of the test score is first processed by Tnorm. If the score is larger than the threshold, the acoustic feature of the test utterance will be used to modify the GMM of the speaker model. For simplicity, we use the original training utterance and the test utterance to re-train the speaker model.

(7).In system 7, both unsupervised model training and unsupervised score normalization are adopted to improve performance.

\subsection{Experimental results and analysis}

The equal error rate (EER) and the minimum decision cost value (minDCF) are addressed to evaluate system performance [1]. The EER and minDCF (without Cnorm) of the experiments is shown in Table 1.

From table 1, factor analysis can improve the performance significantly against the baseline GMM-UBM system. Furthermore, after factor analysis processing, the normalization technique can reduce the EER from $6.13 \%$ to $5.08 \%$ (system 2 to system 3). This is because the factor analysis can remove the channel bias, the normalization can remove the intra-speaker bias and enhance the inter-speaker difference. System 4 and system 6 achieve almost the same EER and minDCF. System 4 adopts unsupervised score normalization, system 6 adopts unsupervised model training. The computation load of system 4 is much less than system 
6. This is the advantage of the unsupervised score normalization, making it useful in on-line system application. System 5 can also improve the performance to some extent.

Finally, the best performance can be obtained if both unsupervised model training and unsupervised score normalization are used as in recognition system 7.

Table 1. The performance on the NIST 2006 SRE core test

\begin{tabular}{|c|l|c|c|}
\hline & \multicolumn{1}{|c|}{ System description } & MinDCF & EER \\
\hline 1 & GMM-UBM,2048 GMM,Tnorm & 0.047 & $9.02 \%$ \\
\hline 2 & $\begin{array}{l}\text { GMM_UBM, 512 GMM, factor } \\
\text { analysis. }\end{array}$ & 0.031 & $6.13 \%$ \\
\hline 3 & $\begin{array}{l}\text { GMM_UBM, 512 GMM, factor } \\
\text { analysis, TZNorm }\end{array}$ & 0.025 & $5.08 \%$ \\
\hline 5 & $\begin{array}{l}\text { GMM_UBM, 512 GMM, factor } \\
\text { analysis, TNorm, unsupervised } \\
\text { score normalization with double } \\
\text { Gauss modeling. }\end{array}$ & $\begin{array}{l}\text { analysis, TNorm, unsupervised } \\
\text { score normalization with } \\
\text { threshold 3.5. }\end{array}$ & 0.023 \\
\hline 6 & $\begin{array}{l}\text { GMM_UBM, 512 GMM, factor } \\
\text { analysis, TNorm, unsupervised } \\
\text { model training }\end{array}$ & 0.022 \\
\hline 7 & $\begin{array}{l}\text { GMM_UBM, 512 GMM, factor } \\
\text { analysis, TNorm, unsupervised } \\
\text { model training, unsupervised } \\
\text { score normalization with double } \\
\text { Gauss modeling. }\end{array}$ & $4.81 \%$ \\
\hline
\end{tabular}

\section{CONCLUSION}

The unsupervised mode is very useful in on-line speaker recognition. Through unsupervised mode, the speaker model can become more and more stable. The speaker model includes GMM model and normalization parameters. We propose the unsupervised score normalization in this paper. The unsupervised score normalization can be treated as a dynamic Znorm, but the unsupervised score normalization can get better performance than the ZTnorm. Furthermore, the unsupervised score normalization can be combined with unsupervised model training to improve the performance.

\section{REFERENCES}

[1]. NIST, The NIST Year 2006 Speaker Recognition
Evaluation Plan, Avail at: http://www.nist.gov/speech /tests/spk/2006/sre-06_evalplan-v9.pdf

[2]. Claudio Vair, Daniele Colibro, Fabio Castaldo, et al, Loquendo-Politecnico diTorino's 2006 NIST Speaker Recognition Evaluation System, Proc. INTERSPEECH 2007:1238 1241

[3]. P. Maťejka, L. Burget, P. Schwarz, O. Glembek, et al, STBU System for the NIST 2006 Speaker Recognition Evaluation, Proc. ICASSP 2007,(4):221 224

[4]. A. Preti1,J.-F. Bonastre1,D. Matrouf, Confidence measure based unsupervised target model adaptation for speaker Verification, Proc. INTERSPEECH 2007: 754 757

[5]. Fr 'ed' eric Bimbot, Jean-Franc, ois Bonastre: A Tutorial on Text-Independent Speaker Verification, EURASIP Journal on Applied Signal Processing 2004,4: 430-451

[6]. Fabio Castaldo,Daniele Colibro,Emanuele Dalmasso, Compensation of Nuisance Factors for Speaker and Language Recognition, IEEE Transactions on Audio, Speech and Language Processing, Sept. 2007,15(7):1969-1978

[7]. P. Kenny, M. Mihoubi, and P. Dumouchel, New MAP Estimators for Speaker Recognition., Proc. EUROSPEECH 2003:2964-2967.

[8]. Robbie Vogt, Sridha Sridharan, experiments in session variability modeling for speaker verification, Proc ICASSP 2006:897 900

[9]. Douglas A. Reynolds, Thomas F. Quatieri and Robert B. Dunn. Speaker verification using adapted Gaussian mixture models. Digital Signal Processing, Academic Press, 2000,10: 19-41 